\title{
Isotope effect of hydrogen release in metal/oxide/n-silicon tunneling diodes
}

\author{
C.-H. Lin, F. Yuan, B.-C. Hsu, C.W. Liu * \\ Department of Electrical Engineering, Graduate Institute of Electronics Engineering, National Taiwan University, Taipei, Taiwan, ROC
}

Received 2 July 2001; accepted 18 November 2002

\begin{abstract}
The metal/oxide/n-silicon tunneling diodes with hydrogen (deuterium) passivated $\mathrm{Si} / \mathrm{SiO}_{2}$ interface are stressed under hole-injection conditions to investigate the mechanism of gate oxide degradation. Although the isotope effect on soft breakdown was previously observed in the deuterium-annealed metal/oxide/p-silicon devices, no isotope effect on the oxide soft breakdown was observed in the metal/oxide/n-silicon devices. However, the time evolution of electroluminescence indeed shows the isotope effect on the interface states density at the $\mathrm{Si}_{\mathrm{SiO}}$ interface of the metal/oxide/nsilicon devices. This suggests that there is an isotope effect on the hydrogen (deuterium)-release at $\mathrm{Si} / \mathrm{SiO}_{2}$ interface under hole current stress from the gate electrodes, but the released hydrogen moves to the bulk Si (not oxide) due to the direction of the electric field. This can explain that the isotope effect is observed in the electroluminescence measurement, but not in the soft breakdown measurement. The hydrogen released to the bulk Si is not responsible for the soft breakdown, and the tunneling-hole-induced traps in the oxide may be responsible.
\end{abstract}

(c) 2003 Published by Elsevier Science Ltd.

Keywords: Ultrathin oxide; MOS; Deuterium; Degradation; Hole trapping

\section{Introduction}

The time-dependent degradation of metal-oxide-silicon (MOS) devices due to current stress has been extensively studied since the early 1980s [1], and the reliability of ultrathin oxide becomes an important issue for future ultralarge scale integration technology due to the large gate leakage current. It is generally believed that gate oxide degrades after a critical density of electron traps has been built-up in bulk oxide [2]. However, the mechanism of the trap generation is still a concerned issue these years. DiMaria and Cartier proposed that the trap creation was related to the $\mathrm{Si} / \mathrm{SiO}_{2}$ interface hydrogen release (HR) by injected hot electrons [3], i.e., "HR model". The reliability improvement by replacing hydrogen with deuterium at the $\mathrm{Si} / \mathrm{SiO}_{2}$ interface using

\footnotetext{
${ }^{*}$ Corresponding author. Tel.: +886-2-2363-5251x515; fax: +886-2-2363-8247.

E-mail address: chee@cc.ee.ntu.edu.tw (C.W. Liu).
}

post-metallization anneal supports this model [4]. The improvement is due to the strong coupling between Si-D bending mode $\left(460 \mathrm{~cm}^{-1}\right)$ and transverse optical phonons in bulk Si $\left(463 \mathrm{~cm}^{-1}\right)$ [5-7]. However, the anode/ hot hole injection model was imposed recently because no improvement in oxide reliability was observed in the devices with deuterium passivated $\mathrm{Si} / \mathrm{SiO}_{2}$ interface under Fowler-Nordheim stress [8,9]. We have proposed that the oxide degradation is related to the HR model under low injected electron energy and high current density stress condition in n-channel MOS tunneling diodes [10]. In this letter, we investigate the degradation of the hydrogen- and deuterium-treated p-channel MOS (PMOS) tunneling diodes under positive gate voltage stress. The experimental results reveal that the deuterium isotope effect is not observed on the oxide soft breakdown, while the isotope effect is indeed observed on the interface states density $\left(D_{\mathrm{it}}\right)$ monitored by electroluminescence intensity. However, the breakage of the $\mathrm{Si}-\mathrm{H}$ bonds does not contribute to the trap formation in the oxide due to that the released hydrogen moves into the bulk Si. 


\section{Experiments}

The ultrathin gate oxide of the PMOS tunneling diode used in this study was grown by rapid thermal oxidation (RTO) on n-type $\mathrm{Si}$ at $900{ }^{\circ} \mathrm{C}$. The gas flows were $500 \mathrm{sccm}$ nitrogen and $500 \mathrm{sccm}$ oxygen at the pressure of 100-250 mbar. Before oxidation, the sample was cleaned by a HF dip. An in situ deuterium prebake at $900{ }^{\circ} \mathrm{C}$ for $2 \mathrm{~min}$ was performed before the growth of the RTO. After the growth of the ultrathin oxide, the sample was in situ annealed in deuterium and nitrogen for $10 \mathrm{~min}$ each at $900{ }^{\circ} \mathrm{C}$. This yields a deuterium concentration of $2 \times 10^{20} \mathrm{~cm}^{-3}$ in oxide measured by the secondary ion mass spectroscopy using the same growth condition (Fig. 1 of Ref. [11]). The $\mathrm{H}_{2}$-treated samples were processed with the same procedure except replacing deuterium by hydrogen. The wafer temperature was measured by a pyrometer with a close loop control. The thickness of oxide was measured by ellipsometry. The resistivity of the $100 \mathrm{~mm}$ n-type $(100)$ wafers is $1-10$ $\Omega \mathrm{cm}$. PMOS diodes have Al gate electrodes with circular areas defined by photolithography. In this experiment, the reliability measurement was carried out using an HP 4156A semiconductor parameter analyzer.

\section{Results and discussion}

Fig. 1 shows the time evolution of gate current for $\mathrm{H}_{2}$-treated PMOS tunneling diodes with oxide thickness of $2.7 \mathrm{~nm}$ under constant voltage stress (CVS) at $V_{\mathrm{g}}=2$ $\mathrm{V}$. The area size of the device is $3 \times 10^{-4} \mathrm{~cm}^{2}$. At positive gate bias, electrons tunnel from the silicon substrate to gate electrodes, and holes tunnel from gate electrodes to silicon substrate. Note that the electron tunneling from

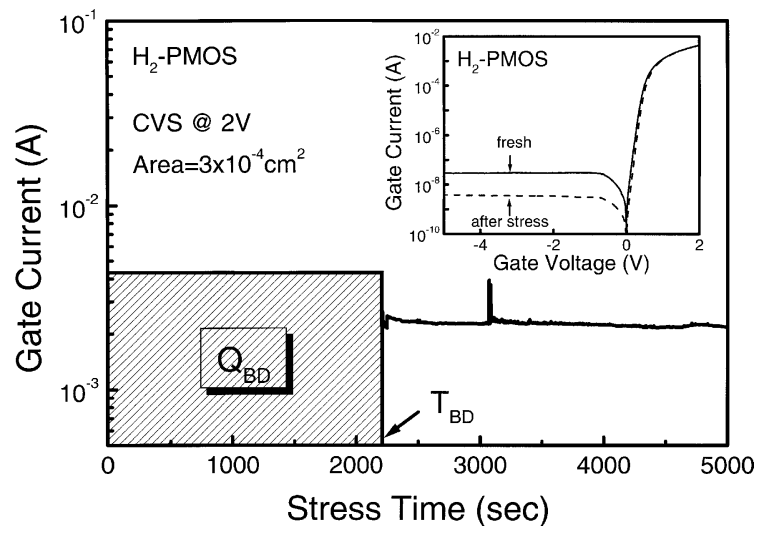

Fig. 1. Time evolution of the gate current for $\mathrm{H}_{2}$-treated PMOS tunneling diodes with oxide thickness of $2.7 \mathrm{~nm}$ under CVS at $V_{\mathrm{g}}=2 \mathrm{~V}$. The inset is the current-voltage curves before and after stress.
Si to $\mathrm{Al}$ cannot damage the $\mathrm{Si} / \mathrm{SiO}_{2}$ interface, since there is no excess energy of electrons at the $\mathrm{Si} / \mathrm{SiO}_{2}$ interface. The device reveals soft breakdown after $\sim 2200$ s stress, which indicates that some traps are built up in the bulk oxide due to the injected holes [13]. There is an apparent disparity between the current-voltage $(I-V)$ curves of the devices before and after stress (the inset of Fig. 1). Compared with the results of the $\mathrm{H}_{2}$-treated devices, similar phenomena are observed in $\mathrm{D}_{2}$-treated devices under the same stress condition. No deuterium isotope effect on soft breakdown is observed in the $\mathrm{H}_{2}$ - and $\mathrm{D}_{2}$ treated PMOS tunneling diodes under hole injection stress.

To confirm the observed results, more than 10 devices are measured to obtain the statistical data. Fig. 2 shows the Weibull plot of the charge to breakdown $\left(Q_{\mathrm{BD}}\right)$ characteristics for both the $\mathrm{H}_{2}$ - and the $\mathrm{D}_{2}$-treated PMOS tunneling diodes with oxide thickness of 2.7 and $2.8 \mathrm{~nm}$, respectively, under CVS at $V_{\mathrm{g}}=2 \mathrm{~V}$. The area of the devices is $3 \times 10^{-4} \mathrm{~cm}^{2}$. The $Q_{\mathrm{BD}}$ is calculated from the integral of the gate tunneling current and the time-to-breakdown $\left(T_{\mathrm{BD}}\right)$ as shown in Fig. 1 (labeled as " $Q_{\mathrm{BD}}$ "). Both $\mathrm{H}_{2}$ - and $\mathrm{D}_{2}$-treated devices have similar $Q_{\mathrm{BD}}$ distribution. No improvement of oxide reliability is observed in the $\mathrm{D}_{2}$-treated devices. Based on the HR model, the isotope effect is due to the strong coupling between $\mathrm{Si}-\mathrm{D}$ bond bending mode and silicon optical phonon states, and as a result, the $\mathrm{Si}-\mathrm{D}$ bonds become more difficult to be broken by injected electrons than $\mathrm{Si}-$ $\mathrm{H}$ bonds $[5,10]$. The isotope effect on soft breakdown is not observed in our experiments. Therefore, in the PMOS tunneling diodes, the degradation of the gate oxide may be dominated by other mechanisms.

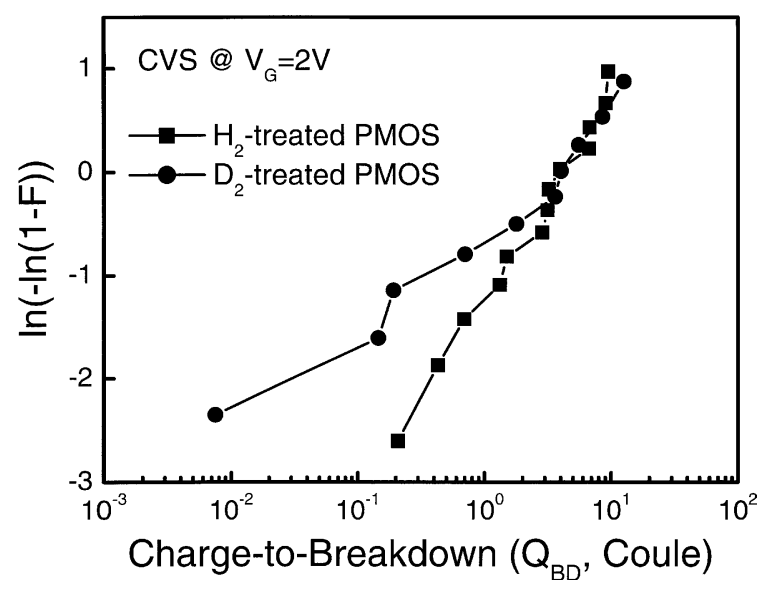

Fig. 2. The Weibull plot of the charge to breakdown $\left(Q_{\mathrm{BD}}\right)$ characteristics for the $\mathrm{H}_{2}$ - and the $\mathrm{D}_{2}$-treated PMOS tunneling diodes under CVS at $V_{\mathrm{g}}=2 \mathrm{~V}$. No deuterium isotope effect is observed. 
One speculative mechanism is the HR from the Al gate. It is well known that the $\mathrm{Al}$ gates contain high concentration of hydrogen. The injected electrons from Si to Al gate may cause the HR from the gate, which will lead to the degradation of oxide. There will be no difference between the reliability performance of the $\mathrm{H}_{2}-$ and $\mathrm{D}_{2}$-treated devices if the HR from the gate becomes the dominant mechanism. The other possible mechanism is direct hole trapping in the oxide. Two kinds of current components exist in the PMOS tunneling diodes. The electron current tunnels from Si conduction band to $\mathrm{Al}$ and the hole current tunnels from $\mathrm{Al}$ to $\mathrm{Si}$ valence band. The hole tunneling from $\mathrm{Al}$ to $\mathrm{Si}$ can break the $\mathrm{Si}-$ $\mathrm{O}$ bond or $\mathrm{Si}-\mathrm{H}$ bond [12] or be trapped in the bulk oxide by oxygen vacancy (O vacancy) $[13,14]$ with the formation of the interface states and bulk tarp, respectively. While the electron tunneling from Si to $\mathrm{Al}$ cannot damage the $\mathrm{Si} / \mathrm{SiO}_{2}$ interface, since there is no excess electron energy at the $\mathrm{Si} / \mathrm{SiO}_{2}$ interface. By theoretical calculation, Yokozawa et al. have proposed that the $\mathrm{O}$ vacancy terminated with hydrogen initially can change its structure to be a new electron trap after capturing injected holes [13,14]. Therefore, if the injected holes have enough energy to break the $\mathrm{Si}-\mathrm{H}$ bonds in the bulk oxide (hole trapped by $\mathrm{O}$ vacancy), the generation of traps will contribute to the leakage currents in oxide film [14], which will lead to the degradation of oxide. However, these models are speculative and still under investigation.

To further investigate the defect formation in the PMOS tunneling diodes, the light emission intensity at bandgap energy is measured [15]. The band-edge light emission can be observed when the oxide roughness [16] and phonons [17] can provide the necessary momentum. Fig. 3 shows the mechanism of light emission of the PMOS tunneling diodes. The device is biased at accumulation region, i.e., the positive voltage on the gate electrode. The holes tunnel from gate to the n-Si substrate, recombine with the electrons in the accumulation region, and then the light is emitted (radiative recombination). However, if the holes recombine with the electrons via the interface states, the emission intensity at bandgap energy decreases (non-radiative recombination). Therefore, we can investigate the variation of the $D_{\text {it }}$ by monitoring the time evolution of the emission intensity. Note that it is difficult to get the information of the $D_{\mathrm{it}}$ in the MOS diode with ultrathin oxide from capacitance-voltage measurement due to the significant leakage current. The time evolution of the emission intensity at the peak of the spectra was measured in the Fig. 4. The normalized emission intensity at the peak of the spectra for the $\mathrm{D}_{2}$-treated device decreases only $\sim 9 \%$ after 10,000 s constant current stress at $100 \mathrm{~mA}$. While, the normalized emission intensity for hydrogen-treated device decreases $\sim 33 \%$ after $10,000 \mathrm{~s}$ stress. For the $\mathrm{H}_{2}$ treated device, the hydrogen bounded at interface $(\mathrm{Si}-\mathrm{H}$

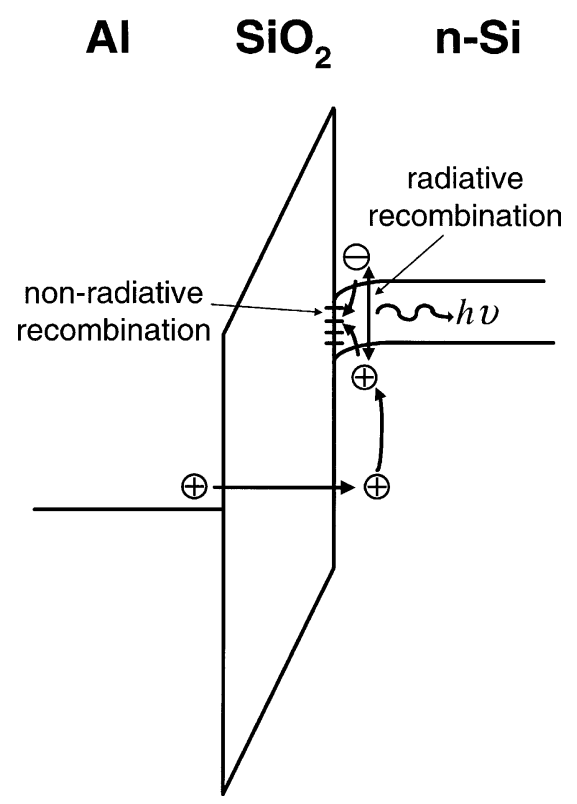

Fig. 3. The mechanism of light emission of the PMOS tunneling diodes.

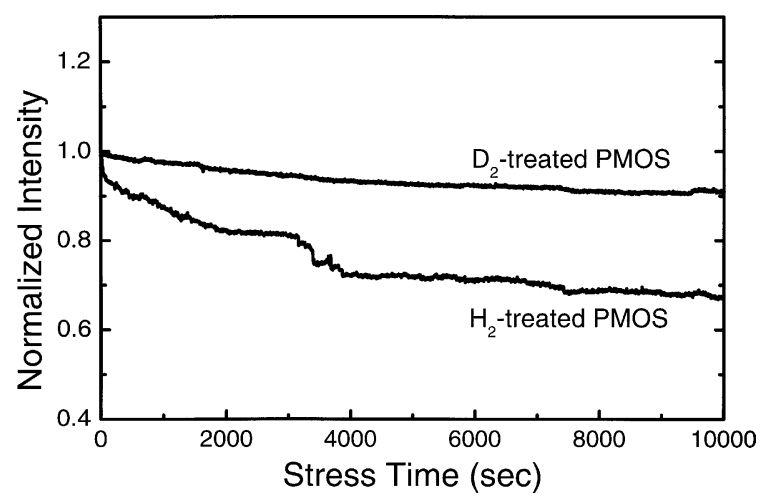

Fig. 4. Time evolutions of the emission intensity at peak for both $\mathrm{H}_{2}$ - and the $\mathrm{D}_{2}$-treated PMOS tunneling diodes under constant current stress at $100 \mathrm{~mA}$.

bonds) was more easily released due to the injected holes than the deuterium. Therefore, the $D_{\text {it }}$ increases and the non-radiative recombination rate via interface states increases with the stress time. The deuterium isotope effect is observed on $D_{\text {it }}$, indicating that there must be other origins of trap formation in the oxide of the PMOS device rather than the HR model under hole injection stress.

The speculative mechanism of the trap formation in the oxide of the PMOS device under hole injection stress is shown in Fig. 5. The hole tunneling from $\mathrm{Al}$ to $\mathrm{Si}$ can break the $\mathrm{Si}-\mathrm{H}$ bond or be trapped in the bulk oxide by 


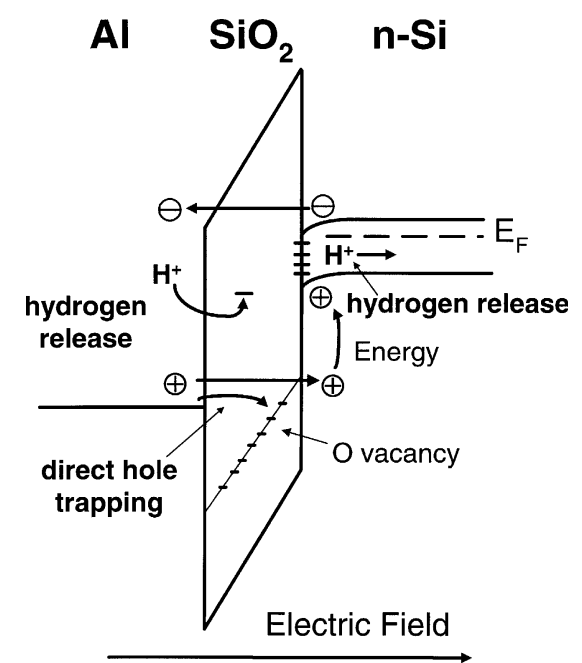

Fig. 5. The speculative mechanism of the trap formation in the oxide of the PMOS device under hole injection stress.

the oxygen vacancy (direct hole trapping) with the formation of the interface states and oxide traps, respectively. Since the released hydrogen moves forward into the bulk Si, not into the oxide, due to the direction of electric field, the released hydrogen cannot contribute to the trap formation in the oxide. The injected electrons from Si to Al gate may cause the HR from the gate. The origin of oxide traps may come from the direct hole trapping in the oxide or the HR from the Al gate, which induce the oxide soft breakdown. Therefore, no deuterium isotope effect on soft breakdown is observed in the hole injection condition.

\section{Conclusions}

In conclusion, the degradation mechanism of PMOS tunneling diodes under hole injection is investigated. The soft breakdown has no isotope effect, but the electroluminescence has. The soft breakdown may be caused by the trap formation in oxide induced by the direct hole trapping in the oxide or the HR from the Al gate. The released hydrogen from the breakage of $\mathrm{Si}-\mathrm{H}$ bonds at $\mathrm{Si} / \mathrm{SiO}_{2}$ interface cannot contribute to the formation of oxide traps due to the direction of the electric field.

\section{Acknowledgements}

This work is supported by National Science Council (89-2218-E-002-082 and 89-2218-E-002-054), and Institute of Applied Science and Engineering Research, Academia Sinica, ROC.

\section{References}

[1] Maserijian J, Zamani N. Behavior of the $\mathrm{Si} / \mathrm{SiO}_{2}$ interface observed by Fowler-Nordheim tunneling. J Appl Phys 1982;53(1):559-67.

[2] Degraeve R, Groeseneken G, Bellens R, Ogier JL, Depas M, Roussel PJ, et al. New insights in the relation between electron trap generation and the statistical properties of oxide breakdown. IEEE Trans Electron Dev 1998;45(4): 904-11.

[3] DiMaria DJ, Cartier E. Mechanisms for stress-induced leakage currents in thin silicon dioxide films. J Appl Phys 1995;78(6):3883-94.

[4] Lyding JW, Hess K, Kizilyalli IC. Reduction of hot electron degradation in metal oxide semiconductor transistors by deuterium processing. Appl Phys Lett 1996;68(8): 2526-8.

[5] Van de Walle CG, Jackson WB. Comment on "Reduction of hot electron degradation in metal oxide semiconductor transistors by deuterium processing". Appl Phys Lett 1996; 69(16):2441-3.

[6] Hess K, Kizilyalli IC, Lyding JW. Giant isotope effect in hot electron degradation of metal oxide silicon devices. IEEE Trans Electron Dev 1998;45(2):406-16.

[7] Wei JH, Sun MS, Lee SC. A possible mechanism for improved light-induced degradation in deuterated amorphous-silicon alloy. Appl Phys Lett 1997;71(11):1498-500.

[8] Wu J, MacDonald RB, Li E, Tao J, Tracy B, Fang P. Anode hole injection versus hydrogen release: the mechanism for gate oxide breakdown. IRPS Tech Dig 2000:27-32.

[9] Esseni D, Bude JD, Selmi L. Deuterium on interface states and SILC generation in CHE stress conditions: a comparative study. IEDM Tech Dig 2000:339-42.

[10] Lin CH, Lee MH, Liu CW. Correlation between Si-H/D bond desorption and injected electron energy in metaloxide-silicon tunneling diodes. Appl Phys Lett 2001;78(5): 637-9.

[11] Liu CW, Lin CH, Lee MH, Chang ST, Liu YH, Chen MJ, et al. Enhanced reliability of electroluminescence from metal-oxide-silicon tunneling diodes by deuterium incorporation. Appl Phys Lett 2001;78(10):1397-9.

[12] Chen Z, Garg P, Singh V, Chetlur S. Role of holes in the isotope effect and mechanisms for the metal-oxide-semiconductor device degradation. Appl Phys Lett 2001;79(2): 212-4.

[13] Yokozawa A, Oshiyama A, Miyamoto Y, Kumashiro S. Oxygen vacancy with large lattice distortion as an origin of leakage currents in $\mathrm{SiO}_{2}$. IEDM Tech Dig 1997:703-6.

[14] Yokozawa A, Miyamoto Y. First-principles exploration of possible trap terminators in $\mathrm{SiO}_{2}$. Appl Phys Lett 1998; 73(8):1122-4.

[15] Liu CW, Lee MH, Chen MJ, Lin IC, Lin CF. Roomtemperature electroluminescence from electron-hole plasmas in the metal-oxide-silicon tunneling diodes. Appl Phys Lett 2000;76(12):1516-8.

[16] Liu CW, Lee MH, Chen MJ, Lin CF, Chern MY. Roughness-enhanced electroluminescence from metal silicon oxide tunneling diodes. Electron Dev Lett 2000;21(12):601-3.

[17] Liu CW, Chen MJ, Lin IC, Lee MH, Lin CF. Temperature dependence of the electron-hole-plasma electroluminescence from metal-oxide-silicon tunneling diodes. Appl Phys Lett 2000;77(18):1111-3. 